\title{
Factors Related To Non-Adherence To Lifestyle Modification In Patients With Diabetes Mellitus Type 2 At Harare Central Hospital
}

\author{
LynneteChilala Mukonka $^{1}$, Doreen Mukona ${ }^{2}$,Matilda Zvinavashe ${ }^{3}$, \\ Babill Stray-Pedersen ${ }^{4}$ Augustine Ndaimani ${ }^{5}$ Maxwell Mhlanga ${ }^{6}$ \\ 1, 2, 3,5,6 University of Zimbabwe College of Health Sciences Department of Nursing Science \\ ${ }^{4}$ Division of Women, Rikshospitalet, Institute of Clinical Medicine, University of Oslo, Norway
}

\begin{abstract}
Lifestyle modification is an important component of long-term management of patients with diabetes mellitus. The objectives of the cross-sectional study were to measure the level of adherence to lifestyle modification, to identify the factors influencing adherence to lifestyle modification, to measure the prevalence of diabetes complications among patients with type 2 diabetes mellitus. The sample comprised 119 participants who were diabetes mellitus type 2 patients registered at the study site, aged 18 years and above, could converse in English or Shona, and had been diagnosed within a period of 2 years. They were selected using simple random sampling with replacement. Data was collected through an interviewer administered questionnaire.. Adherence to lifestyle modification was low (7.56\%). Factors influencing adherence to lifestyle modification; for diet they include, lack of money (21.01\%), attending functions (15.13\%), lack of satiety (12.61\%), tempting foods (12.61\%); for exercises they include, illness (26.89\%), lack of time (18.49\%), fatigue (15.13\%) and no education (10.92\%). The most common complications were retinopathy (79.83\%), peripheral neuropathy (78.99\%) and sexual dysfunction (44.54\%). A weak negative linear relationship was shown between adherence to lifestyle modification and the total number of diabetes complications $(r=-0.1398, p=0.1295)$ but was not significant thus linear regression was not done.
\end{abstract}

Keywords:adherence, diabetes mellitus type 2, lifestyle modification

\section{Introduction}

Globally, in 2013, the prevalence of diabetes was $8.3 \%$ and in Africa, it was 5.7\%. It is projected that the numbers will double by the year 2035. In Zimbabwe, the prevalence of diabetes was 8.8\% [1] Health costs due to diabetes amounted to US \$ 612billion worldwide. Worldwide 1 person dies every 7 seconds from diabetes, and 50 percent of the deaths are occurring in those below the age of 60 [2]. The age of onset of diabetes is decreasing especially for type 2 diabetes because of the growing epidemic of obesity.

Diabetes mellitus type 2 is a type of diabetes mellitus formerly referred to as non-insulin-dependent or adult- onset diabetes. It is caused by the body's ineffective use of insulin [3]. According to the Centre for Disease Control (2014) [4], $95 \%$ of diabetic patients have type 2 diabetes mellitus.Patients with type 2 diabetes mellitus are at risk of complications such as diabetic retinopathy, neuropathy, vascular damage and nephropathy [5]. These complications can be minimized by maintaining normal blood glucose level of between 3 to 6 mmol/L. However, Essential Drugs List of Zimbabwe (2013)[6] recommends that the initial management for both obese and normal weight patients includes lifestyle modifications such as weight reduction (for obese patients) and diet change for both obese and normal weight patients. Medications become necessary only when there is poor glycemic control.

The consequences for poor adherence include complications of diabetes, high costs of treatment especially when there are long-term complications [7]. The cost is particularly huge for developing countries [8] and quality of life of the clients is also affected [8].Lifestyle modification is crucial for long-term management of patients with type 2 diabetes. It combines diet and exercise with cognitive behavioral strategies. Cognitive behaviour therapy is based on the behaviorists' learning theory, it is believed that excessive eating and low activity levels are behaviours that are learnt and thus can be changed [9].The focus is on teaching clients on how to change their eating and exercise habits through individual or group sessions. Lifestyle modification based on behavioral therapy is an important and effective strategy in the management of diabetes mellitus [9]. The strategies include use of self-monitoring and goal setting, controlling the stimuli and change of eating style and habits as well as reinforcement of the desired behaviour. Focus should also be on coping skills such as stress management, and utilization of social support [10]. At Harare Central Hospital in Harare, continuous efforts are being made to instill skills on lifestyle modification, among type 2 diabetic patients. Patients are given health education on diet, exercise, self-monitoring of blood glucose levels, smoking and alcohol intake cessation, and stress management. 
Informal interactions with type 2 diabetic patients show that the patients were finding it difficult to adjust to the requisite lifestyle modifications. This study sought to identify factors related to non- adherence to lifestyle modification among patients with type 2 diabetes. At Harare Central Hospital Diabetic Clinic an average of 200 patients are seen per month. Of all the diabetic patients with type 2 diabetes mellitus seen in the diabetic clinic, $98.1 \%$ are on drug therapy while the remaining $1.9 \%$ is on diet control alone. The high number of patients on drug therapy might imply that they failed to maintain normal glycaemic levels using lifestyle modification alone.Anecdotal evidence shows that type 2 diabetic patients are failing to adhere to lifestyle modification. The non-adherence problem is so common that the patients who could be managed on diet are quickly put on hypoglycaemic agents due to failure to achieve glycaemic control. As a result, the number of patients with type 2 diabetes who are on drug therapy is relatively high compared to those on diet control.

Failure to adhere to lifestyle modification affects not only the patient but the family, the hospital, and the nation as a whole. The patient as indicated above will develop complications earlier than they would if they adhered to lifestyle modification [11]. They may even be admitted into hospital for stabilization of blood glucose level or management of complications. When a family member is ill the family is disturbed in various ways. Some patients maybe the breadwinners for their families and the illness may rob them of productive time. Some may not have someone to look after their young children while they are admitted in hospital. Type 2 diabetes mellitus is considered a serious and costly disease [12]. Health costs due to diabetes amounted to US \$ 612billion worldwide. Worldwide 1 person dies every 7 seconds from diabetes, and 50 percent of the deaths are occurring in those below the age of 60 [2]

According to the International Diabetes Federation Africa (IDF), (2014), Zimbabwe, in 2014, had 605 100 cases of diabetes and the prevalence of diabetes in adults was $8.5 \%$. The cost per person with diabetes was 58.6 United State dollars. 16704 deaths were attributed to diabetes [13]. The cost is very high and thus requires measures to reduce these costs especially in a developing country like Zimbabwe, where the majority live below the poverty datum line of $\$ 511.00$ as of January 2014 [14] This study therefore sought to identify the factors related to non-adherence to lifestyle modification among these patients. When identified, measures can be instituted to minimize the factors and thus promote adherence to lifestyle modification interventions. This information from clients with type 2 diabetes mellitus will also be essential in delaying the onset of complications[11].Nola Pender's Health Promotion Model was used as the guiding framework for this study. It assists in understanding major determinants of health behaviors as a basis for promoting healthy lifestyles. The model looks at factors which affect health behavior. It focuses on individual characteristics and experiences, behavior specific cognitions and affect and behavioral outcomes [15]

\section{Materials And Methods}

A cross-sectional survey was conducted at Harare Central Hospital's diabetic clinic. Simple random sampling with replacement was used to select 119 diabetes mellitus type 2patients, aged 18 years and above who had been diagnosed within 2 years. The patients could converse in English or Shona. Patients who were critically ill, mentally and institutionalized clients were excluded because of their compromised autonomy. The Dobson formula was used to calculate the sample size The sample size was calculated using the Dobson formula that is;

$$
\mathrm{n}=\frac{Z^{2} P(1-P)}{d^{2}}
$$

where $\mathrm{n}$ is the minimum number of participants required for the study, $\mathrm{Z}$ was the $\mathrm{Z}$ statistic for the level of confidence, $\mathrm{P}$ was the expected prevalence and $\mathrm{d}$ was the precision. The prevalence of diabetes in Zimbabwe that is $8.5 \%$ [1] was used. A $5 \%$ level of significance was used which was also the value of $\mathrm{d}$ and thus $\mathrm{Z}$ was equal to 1.96.Data was collected using an interviewer administered questionnaire which we developed making reference to literature. It was divided into 3 sections. Section A was on demographic data, this section asked about the study participant's age, sex, and marital status, level of education, employment status, religion, income and place of residence. The time of diabetes diagnosis, the treatment they were put on initially and the current treatment were asked for. The client's height, weight, body mass index, waist circumference, blood glucose levels and latest glycosylated hemoglobin levels were recorded in this section.

The extent to which the participants adhere to lifestyle modification that is diet and exercise, smoking and drinking habits, stress management and self-monitoring of blood glucose levels recommendations were assessed in Section B. The responses were graded to allow for measurement of level of adherence. The participants were also asked to indicate reasons for non-adherence if they indicated that they sometimes or never adhered to diet or exercise recommendations. Section $\mathrm{C}$ was for diabetes related complications. The participants were asked to indicate the complications that they were experiencing or had experienced from a given list of complications.Ethical approval was sought from the Joint Research Ethics Committeefor The University of Zimbabwe, College of Health Sciences and Parirenyatwa Group of Hospitals and the Medical Research Council of Zimbabwe (MRCZ) and from the Harare Central Hospital ethics committee. A signed informed consent was obtained from each participant. 
Data was analysed using descriptive statistics that is frequencies, means, modes, medians and percentages, and inferential statistics that is the Pearson's correlation test and regression analysis. Stata Version 13 was used for analysis.

\section{Patient characteristics}

\section{Results And Findings}

Table 1 below shows thw demographic characteristics of the study participants. The ages of the study participants ranged from 31 to 88 years, with mean age of 55.5years (SD14.4). Eighty-five (71\%) were females, and $93(78 \%)$ were urban residents. Sixty-seven $(55.30 \%)$ went to school up to primary level. Seventy-nine $(66.39 \%)$ were unemployed and $109(91.6 \%)$ had a monthly family income below US\$600. Ninety-six (80.67) had no medical insurance.

Table 1-Sample Demographics (1) $(\mathrm{n}=119)$

\begin{tabular}{|c|c|c|}
\hline Variable & Frequency & Percent \\
\hline \multicolumn{3}{|l|}{ Age in years } \\
\hline $30-39$ & 10 & 8.40 \\
\hline $40-49$ & 25 & 21.01 \\
\hline $50-59$ & 34 & 28.57 \\
\hline $60-69$ & 32 & 26.89 \\
\hline $70-79$ & 13 & 10.92 \\
\hline $80-89$ & 5 & 4.20 \\
\hline \multicolumn{3}{|l|}{$\underline{\operatorname{Sex}}$} \\
\hline Male & 34 & 28.57 \\
\hline Female & 85 & 71.43 \\
\hline \multicolumn{3}{|l|}{ Marital status } \\
\hline Married & 78 & 65.55 \\
\hline Single & 6 & 5.04 \\
\hline Widowed & 31 & 26.05 \\
\hline Divorced & 4 & 3.36 \\
\hline \multicolumn{3}{|l|}{ Religion } \\
\hline Christianity & 118 & 99.16 \\
\hline No religion & 1 & 0.84 \\
\hline \multicolumn{3}{|l|}{ Place of residence } \\
\hline Urban & 93 & 78.15 \\
\hline Rural & 26 & 21.85 \\
\hline
\end{tabular}

Table 2 below shows the sample health history that is the duration of diabetes mellitus diagnosis in months, initial and current treatment regimen that the participants were on for the treatment of diabetes.

Table 2: Sample Health History, $(\mathrm{n}=119)$

\begin{tabular}{|l|r|c|}
\hline Variable & Frequency & Percent \\
\hline Duration of DM diagnosis in months & & \\
\hline $0-4$ & 33 & 27.73 \\
\hline $5-9$ & 33 & 27.73 \\
\hline $10-14$ & 22 & 18.49 \\
\hline $15-19$ & 9 & 7.56 \\
\hline $20-24$ & 22 & 18.49 \\
\hline Initial treatment & & \\
\hline Diet only & 19 & 15.97 \\
\hline Oral hypoglycaemic agents & 94 & 78.99 \\
\hline Oral hypoglycaemic agents and insulin & 1 & 0.84 \\
\hline Insulin only & 5 & 4.20 \\
\hline Current treatment & 5 & 4.20 \\
\hline Diet only & 102 & 85.71 \\
\hline Oral hypoglycaemic agents & 6 & 5.04 \\
\hline Oral hypoglycaemic agents and insulin & 6 & 5.04 \\
\hline Insulin only & & \\
\hline
\end{tabular}

Table 3 below shows the sample's anthropometric measurements, which include mean body mass index, obesity classification according to WHO, waist circumference and presence of central obesity. Most of the participants had both general obesity and central obesity. Eighty-three (70\%) were hyperglycaemic on testing their random blood glucose levels, but the glycosylated haemoglobin was within the normal range for most of the participants. 
Table 3: Sample Anthropometric Measurements $(\mathrm{n}=119)$

\begin{tabular}{|l|c|c|}
\hline Variable & Frequency & Percent \\
\hline Body mass index & & \\
\hline$<16$ (severe thinness) & 1 & 0.84 \\
\hline $16-17$ (moderate thinness) & 1 & 0.84 \\
\hline $17-18.5$ ( mild thinness) & 0 & 0.00 \\
\hline $18.5-25$ (normal) & 42 & 35.29 \\
\hline $25-30$ (overweight) & 36 & 30.25 \\
\hline $30-35$ (obese class I) & 29 & 24.37 \\
\hline $35-40$ (obese class II) & 9 & 7.56 \\
\hline$>40$ (obese class III) & 1 & 0.84 \\
\hline Waist circumference & & \\
\hline$<88$ cm (female) (normal) & 14 & 11.76 \\
\hline$<102 \mathrm{~cm}$ (male) (Normal) & 22 & 18.49 \\
\hline$>88 \mathrm{~cm}$ (female) (central obesity) & 71 & 59.66 \\
\hline$>102 \mathrm{~cm}$ (male) (central obesity) & 12 & 10.08 \\
\hline Random blood sugar levels in mmol/L & & \\
\hline$<5$ (hypoglycaemia) & 4 & 3.36 \\
\hline $5-11$ (normal) & 83 & 69.75 \\
\hline$>11$ (hyperglycaemia) & 32 & 26.89 \\
\hline
\end{tabular}

Complications of diabetes mellitus

Table 4 below shows the complications that the participants had experienced or were experiencing. The median number of complications was 2 with an interquartile range of 2-3.

Table 4: Sample complications and adherence score $(\mathrm{n}=119)$

\begin{tabular}{|l|c|c|}
\hline Variable & Frequency & Percent \\
\hline Retinopathy & 95 & 79.83 \\
\hline Peripheral neuropathy & 94 & 78.99 \\
\hline Nephropathy & 12 & 10.08 \\
\hline Sexual dysfunction & 53 & 44.54 \\
\hline Cerebrovascular accident & 10 & 8.40 \\
\hline Peripheral vascular disease & 3 & 2.52 \\
\hline Amputation & 3 & 2.52 \\
\hline Diabetic ketoacidosis & 1 & 0.84 \\
\hline Hyperglycaemia & 0 & 0.00 \\
\hline Hyperosmolar non-ketotic acidosis & 0 & 0.00 \\
\hline Other conditions & 105 & 88.24 \\
\hline
\end{tabular}

Adherence to lifestyle modification

The maximum possible score for adherence to lifestyle modification was 33, the mean score was 19.1 with a standard deviation of 4.7. The median score was 19 with an interquartile range of $17-22$. Table 5 below shows a summary of the total scores for adherence to lifestyle modification for the study participants.

Table 5: Sample total score for adherence to lifestyle modification( $\mathrm{n}=119)$

\begin{tabular}{|l|c|c|}
\hline Score & Frequency & Percentage \\
\hline Very poor & 13 & 10.92 \\
\hline Poor & 97 & 81.51 \\
\hline Good & 9 & 7.56 \\
\hline Very good & 0 & 0.00 \\
\hline & & \\
\hline
\end{tabular}

Factors influencing adherence to lifestyle modification

Table 6 shows the factors influencing adherence to diet modification and adherence to exercises as indicated by the participants.

Table 6: Sample factors influencing adherence to diet modification and exercises $(n=119)$

\begin{tabular}{|l|c|c|}
\hline Variable & Frequency & Percent \\
\hline Factors influencing diet modification & & \\
\hline No Diet education & 8 & 6.72 \\
\hline Dislike some recommended foods & 2 & 1.68 \\
\hline Attending functions & 18 & 15.13 \\
\hline Visiting & 9 & 7.56 \\
\hline Cooking done by others & 13 & 10.92 \\
\hline No money & 25 & 21.01 \\
\hline Lack of satiety & & 12.61 \\
\hline Tempting foods & 15 & 12.61 \\
\hline
\end{tabular}




\begin{tabular}{|l|r|r|}
\hline Failing to adjust & 12 & 10.08 \\
\hline Bad influence from other DM patients & 1 & 0.84 \\
\hline Other illnesses & 1 & 0.84 \\
\hline Factors influencing adherence to exercises & & \\
\hline No education & 13 & 10.92 \\
\hline Fatigue & 18 & 15.13 \\
\hline Laziness & 11 & 9.24 \\
\hline Illness & 32 & 26.89 \\
\hline Lack of time & 22 & 18.49 \\
\hline Forget to exercise & 8 & 6.72 \\
\hline Old age & 1 & 0.84 \\
\hline
\end{tabular}

Relationship between adherence to lifestyle modification and the total number of diabetes complicationsIn order to establish whether there was a relationship between adherence to lifestyle modification and the prevalence of diabetes related complications, Pearson's correlation coefficient test was used to examine the relationship between adherence to lifestyle modification and the total number of diabetes complications. The result was $r=-0.1398$ with a $\mathrm{p}$ value of 0.1295 which was not significant at 0.05 significance level, this indicated a weak negative linear relationship between adherence to lifestyle modification and the total number of complications in patients with type 2 diabetes mellitus attending the Diabetic Clinic at Harare Central Hospital. Table 11 below shows the correlation matrix of adherence to lifestyle and the total number of diabetes complications. Regression analysis was not done because the result of the Pearson's product-moment correlation was not significant.

Table 11: Correlation Matrix of adherence to lifestyle and the prevalence of diabetes related complications. $(\mathrm{n}=119)$

\begin{tabular}{|l|c|}
\hline Variable & $\mathrm{Y}$ \\
\hline & 1.0000 \\
\hline $\mathrm{X}$ & \\
\hline & -0.1398 \\
\hline & \\
\hline$* \mathbf{p}<\mathbf{0 . 0 5}$ & 0.1295 \\
\hline
\end{tabular}

Key: $\mathrm{X}$ - Adherence to lifestyle modification

$\mathrm{Y}$ - Total number of diabetes complications

A multiple regression test was run to predict adherence to lifestyle modification from age, sex, duration of diagnosis, level of education, employment status, current treatment, self-monitoring of blood glucose levels, having diet plan, perception about diet modification, perception about exercises, ability to cope with stressful events and total number of complications. These variables statistically significantly predicted adherence to lifestyle modification, $F(5,113)=40.48, p<.0005, R^{2}=.6417$. All thirteen variables added statistically significantly to the prediction, $p<.05$. factors which showed a significant association with adherence to lifestyle modification were frequency of self-monitoring of blood glucose levels $(\beta 1.837 ; 95 \% \mathrm{CI} 0.727-2.946 ; \mathrm{p}=$ $0.001)$, having a diet plan $(\beta$ 1.74; $95 \%$ CI $0.559-2.930 ; \mathrm{p}=0.004)$, perception about effect of exercise on glycaemic levels ( $\beta 2.533 ; 95 \%$ CI $1.055-4.012 ; \mathrm{p}=0.001)$ and ability to cope with stressful events ( $\beta$ 1.576; $95 \%$ CI $0.458-2.693 ; p=0.006$ ). Table 11 below shows the relationship between lifestyle modification and the confounding variables.

Table 12: Regression Coefficients for Confounding Variables

\begin{tabular}{|l|l|l|l|}
\hline Variable & Coefficient & Confidence Interval & P Value \\
\hline Frequency of SMBG & 1.837 & $0.727-2.946$ & $0.001^{* *}$ \\
\hline Diet plan about effect of & 1.745 & $0.559-2.930$ & $0.004^{* *}$ \\
\hline $\begin{array}{l}\text { Perception about } \\
\text { exercises on glycaemic levels }\end{array}$ & & $1.055-4.012$ & $0.001^{* *}$ \\
\hline Ability to cope with stress & 2.80 & $0.458-2.693$ & $0.006^{* *}$ \\
\hline
\end{tabular}

\section{Discussion}

Sample demographics and health historyThe study sample comprised 119 participants who were selected through simple random sampling with replacement. The sample size is comparable to non-adherence to lifestyle modification study done by Ganiyu et al.1, (2013)[16] which had a sample size of 105 participants. Other studies which had more than 300 participants, used participants from more than one study site [8; 17] . The ages of participants ranged between 31 and 88 years, with a mean age of 56.5 years and modal age of 53 and 67 years. The age range also concurs with literature as diabetes mellitus type 2 is known to be of adult onset, according to Smeltzer\& Bare, (2012) [18] the age of onset is after 30 years of age. This was also seen in 
this study as the lowest age amongst the participants was 31 years, however, there is literature that states that the age of onset of diabetes mellitus type 2 is decreasing with age due to the growing epidemic of obesity [19]. According to Centre for disease Control, (2013), the mean age of diagnosis for diabetes mellitus type 2 is about 54 years. There were $71.43 \%$ female and $28.57 \%$ male participants in the study. Similar studies had proportions of women being greater than the males although with lower percentage of between 50.2 and $58 \%$ [16; 17]. This could be because of differing population distributions among countries [20]. Some studies done in some West African countries showed no significant differences in the prevalence of diabetes between males and females but studies done in South Africa, Cameron and Uganda revealed that more females were affected by diabetes mellitus type 2 than males. Studies done in Ghana, Sierra Leone, Nigeria and rural Tanzania, the opposite was true with higher prevalence seen in males than females [20]. In this current study, both BMI classified obesity and central obesity were more prevalent in females than in males, obesity is one of the risk factors for the development of type 2 diabetes mellitus as it contributes to insulin resistance $[18 ; 20]$. The obesity among the women could have been brought about by poor eating habits, diets high in saturated fats, and sedentary lifestyles as most women in this setting are unemployed or informally employed.

In this study, $78.15 \%$ were resident in urban areas. The burden and prevalence of type 2 diabetes in Sub-Saharan Africa is rising and this has been attributed to the rapid urbanization and lifestyle changes [21;20]. Urbanisation coupled with globalization has contributed to lifestyle changes including eating habits, as most people shun their native foods in preference to western diets which are high in fats. Fast food outlets have spread all over the world [22]. Improved technology, communication and transport systems have contributed to low activity levels as even for the short walkable distance people board commuter omnibuses or drive their own cars [23].The middle and lower income groups are also affected such as those from the rural areas [24;25], as seen in this study were $21.85 \%$ were from rural areas. Worldwide, low and middle-income countries bear the greatest burden of diabetes and other non-communicable diseases [25].

In this study, only $5.04 \%$ were formally employed and $91.60 \%$ earned below $\$ 600$ per month which is below the poverty datum line for Zimbabwe, of $\$ 511.00$ as of January 2014 [14]. In this study, 80.67\% had no medical insurance cover, which is close to the report in the Chronicle dated 4 September 2014, by Ncube, states that $90 \%$ of Zimbabweans have no access to medical aid. The low income status and lack of medical aid cover could thus pose challenges for the diabetic patients as the cost for diabetes care per person with diabetes was 58.6 United State dollars according to IDF, (2014). Lower educational achievements and low income could be associated with the high prevalence of diabetes because of the social inequalities which may limit access to health information and health care [25].

The sample comprised persons diagnosed of diabetes mellitus type 2, within a period of two years, $55.46 \%$ were diagnosed within a period of up to 9 months, this could be an indication of a rising prevalence which could be consistent with the prediction that prevalence of diabetes type 2 is on the increase and that it is expected to double by 2035 [1]. It could be anticipated that the longer the period of diagnosis could strengthen adherence through repeated education and counselling, however in this study, even those with a longer duration of diagnosis had low adherence scores. This was similar to a study where those with a duration of more than 4 years had lower adherence levels [26]. This could be attributed to the factors such as having no money to buy the recommended foods, attending functions, lack of satiety and that cooking was done by other family members; which participants indicated as some of the reasons for non-adherence to diet modification. As for reasons for non-adherence to exercise recommendation; they indicated presence of other illnesses, lack of time, fatigue and laziness among others.

On diagnosis, $78.99 \%$ of the participants were commenced on oral hypoglycaemic agents, $0.84 \%$ and $4.20 \%$ were on both oral hypoglycaemic agents and insulin, and insulin only, respectively; this could indicate late diagnosis and poor glycaemic control such that lifestyle modification on its own would not be effective for glycaemic control. The reasons for varying initial treatment plans, could also be because of differing doctors' views of and experiences with lifestyle modification recommendations in the management of diabetes mellitus type 2 [27]. Reliance on pharmacological interventions may also have been brought about by increased frequency of prescription of other drugs such as anti-hypertensives [28]. Routine screening for diabetes mellitus in the population could assist with delayed onset of complications, reduced cost of medical care and early diagnosis [29]

As for the treatment that they were on during the time of the study, 85.71 were on oral hypoglycaemic agents only, $5.06 \%$ were either on both oral hypoglycaemic agents and insulin or insulin only. The change in treatment regime could also have financial implications on the patients who are of low economic status [7; 8]. According to IDF, (2014) [19] the cost of medical care per person is US\$58.6, which is very high for persons in developing countries. In this study, the body mass index of the study participants showed that $63.02 \%$ were overweight or obese according to the WHO BMI classification. Central obesity is also associated with diabetes mellitus, in this study, $69.74 \%$ had central obesity. The prevalence of type 2 diabetes mellitus is associated with an increase in body mass index (BMI). People who are obese are 20 times more likely to be diabetic compared 
to those with normal weight. For every 3-7 obese persons with diabetes, there is 1 normal weight adult with diabetes. Weight gain during adulthood is also directly correlated with an increased risk of diabetes mellitus type 2 [30]. Obesity is a common risk factor for diabetes, hypertension and cardiovascular diseases and it increases insulin resistance and blood glucose levels thereby complicating the management of diabetes[18; 30]. The high proportion of participants who were obese could be associated with poor adherence to diet and exercise which could assist in weight reduction [27]. There is need to emphasise on lifestyle modification.

The study participants' random blood glucose levels showed that $26.89 \%$ had hyperglycaemia and $3.36 \%$ had hypoglycaemia. These results show poor glycaemic control. The hyperglycaemic states predispose patients to complications as minimal changes in blood glucose levels are responsible for microvascular and macrovascular complications [11; 27]. According to the participants' treatment record books, the test for glycosylated haemoglobin was done only in $47.9 \%$ of the participants; this could have been due to failure to pay for the test as the cost ranges between US\$7 and US\$25. Amongst those who had the test done, $73.68 \%$ had glycosylated haemoglobin level above $6 \%$, indicating poor glycaemic control for a period of 2 to 3 months.

Factors influencing adherence to lifestyle modification

Lack of money to buy the recommended foods (21.01\%), attending functions $(15.13 \%)$, lack of satiety $(12.61 \%)$ and tempting foods being eaten by other family members $(12.61 \%)$ were the most common factors that the participants gave as reasons for failing to adhere to diet modification. The lack of money as mentioned earlier is supported by the low economic status of the participants as indicated by their low monthly income, and employment status. Other factors indicated by the participants included lack of diet education (6.72\%), dislike of recommended foods (1.68\%), visiting relatives $(7.56 \%)$, especially those who were unaware of their dietary requirements, failure to adjust to the recommendations $(10.08 \%)$, bad influence from other diabetic patients, and other illnesses. Other studies indicated age, duration of disease, lack of communicative relationship between the patient and health care providers, health beliefs and perceptions that are incompatible with the recommendations and socio-economic factors (Mumu et al., 2014).

In the study by Mumu et al., (2014) [17], level of education and area of residence affected adherence to diet as those with a higher level of education adhered to diet recommendations. Those from rural areas were adhered to diet recommendations than those from urban or semi-urban. Patients who attended diabetes education classes tend to follow lifestyle modification recommendations, a study done in Hungary also showed similar results, whereby patients who visited their GP monthly adhered to lifestyle modification recommendations [17]. Barriers to adhering to diet also include unwillingness, diet being different from that for the rest of the family and high frequency of social gatherings [31]..

Perez, et al. (2013)[32] also found out that some patients do not adhere to the dietary recommendations because of dislike for foods in the diet plan. This was similar to the finding in the current study as some indicated dislike of the recommended foods especially vegetables. The various factors indicated in this study and other factors from other studies need to be considered in the management of diabetic patients. There is need for health education, individualized and family teaching, adherence monitoring, engagement of treatment buddies, formation of support groups, as well as home visits to promote adherence to lifestyle modification. At the study site they run a series of lectures every Friday, this could have contributed to only $6.7 \%$ siting lack of diet education as a reason for not adhering to diet modification. However considering that the population is of low economic status going to the hospital for lectures could be unaffordable for the patients as well. In this study, factors influencing adherence to exercises included, illness, lack of time, fatigue, lack of education on exercises, laziness, forgetting to exercise and old age. Some of these factors were also indicated in other studies [8; 16; 17. Patients with other illness thus require education on exercises that are permissible with their conditions. Family support and motivation would be beneficial for those who forgot, felt lazy or thought were too old to exercise.Complications of Diabetes Mellitus

Complications of diabetes mellitus are usually present before the diagnosis of the condition is made as minimal changes in blood glucose levels which are responsible for microvascular and macrovascular complications [11]. In this study, retinopathy, peripheral neuropathy and sexual dysfunction were the most common complications experienced by the study participants some even mentioned that they experienced these problems before diagnosis of diabetes mellitus. The complications thus prompt patients to seek treatment. Other complications which were experienced by the study participants were cerebrovascular accident, peripheral vascular disease, and amputation. Chronic hyperglycaemia is associated with retinal blood vessel damage resulting in diabetic retinopathy. The development of diabetic neuropathy is associated with many factors but amongst them is hyperglycaemia which results in glycation end-products. The glycation end products disrupt neuronal integrity and even repair mechanisms [33]). The damage to the small blood vessels and nerves also occurs in sexual organs resulting in various forms of sexual dysfunction, these problems can be lowered by controlling blood glucose levels [34]. Peripheral neuropathy and arteriosclerosis are also associated with diabetic ulcers [35]. Therefore it is essential that blood glucose levels are monitored regularly and corrective measures should be taken. 
Relationship between Adherence to Lifestyle Modification and the Total Number of Diabetes ComplicationsPearson's correlation coefficient test was used to examine the relationship between adherence to lifestyle modification and prevalence of complications. The result was $(r=-0.1398, p=0.1295)$ which was not significant at 0.05 significance level, this indicated a weak negative linear relationship between adherence to lifestyle modification and prevalence of complications in patients with type 2 diabetes mellitus attending the Diabetic Clinic at Harare Central Hospital. This could mean that adherence to lifestyle modification may reduce the occurrence of complications, however the sample size could have affected the correlation coefficient as well as the fact that only one centre was used in this study. It could also be that complications start to occur before the diagnosis is made such that the patient reports to a health care centre with symptoms of the complications. This therefore indicates the need for early screening for diabetes. There are also other factors that are also related to the development of complications such as age, co-morbidities, stressful life events and side effects of drugs.

Multiple regression of other factors which could influence adherence to lifestyle modification, showed that there are many factors which may influence adherence to lifestyle modification, these included frequency of self-monitoring of blood glucose levels, having a diet plan, perception about the effect of exercise on glycaemic levels, and ability to cope with stress. These factors therefore could have affected the significance of the Pearson's correlation result. Frequency of blood glucose level monitoring was shown to be significantly related to adherence to lifestyle modification $(\beta 1.837 ; 95 \% \mathrm{CI} 0.727-2.946 ; \mathrm{p}=0.001)$, this implies that the more frequent one checks their blood glucose levels, the more they are prepared to take measures to ensure that their blood glucose levels remain within normal ranges. Therefore they would adhere to the lifestyle modification recommendations. In this study, $40.34 \%$ did not did not monitor their blood glucose levels and only $5.88 \%$ checked their blood glucose levels pre- and post- prandial. However, because of the costs associated with SMBG, some patients may be unable to frequently check their glycaemic levels. This also emphasizes the need for subsidization of the glucometers, strips and lancet, as well as conducting patient education programs.As discussed before, diet plans form an important component of the management of diabetes mellitus and on analysis the regression coefficient $(\beta 1.74 ; 95 \% \mathrm{CI} 0.559-2.930 ; \mathrm{p}=0.004)$, this means that having a diet plan promotes adherence to the recommended diet. Therefore to promote adherence every patient should have a diet plan with foods that they can afford or is available in their home setting.

In this study, perception about the effect of exercises on glycaemic levels was shown to be related to adherence to lifestyle modification, $(\beta 2.533 ; 95 \%$ CI $1.055-4.012 ; \mathrm{p}=0.001)$. The way one views exercise may influence even what they choose to eat and even the frequency and type of exercise that they do. Positive perception about exercise will generally result in improved adherence to lifestyle modification. Ability to cope with stressful events was seen to be related to adherence to adherence to lifestyle modification, $(\beta 1.576 ; 95 \% \mathrm{CI}$ $0.458-2.693 ; p=0.006$ ). According to the Canadian Diabetic Association, (2013), stress decreases the ability to adhere to lifestyle recommendations. Therefore it is of importance that diabetic patients be taught about stress management and coping skills. Strong social support from family and support groups is also important. A review by Jin, Sklar, Min Sen Oh, \& Chuen Li, (2008) [17], showed that those who have emotional support from fanily, friends and healthcare workers were likely to adhere to recommended therapy.

\section{Conclusion}

Adherence to lifestyle modification was low $(7.56 \%)$. Various factors related to non-adherence to lifestyle modification were identified, for diet they include, lack of money $(21.01 \%)$, attending functions (15.13\%), lack of satiety (12.61\%), tempting foods (12.61\%); for exercises they include, illness $(26.89 \%)$, lack of time $(18.49 \%)$, fatigue $(15.13 \%)$ and no education $(10.92 \%)$. The most common complications were retinopathy (79.83\%), peripheral neuropathy $(78.99 \%)$ and sexual dysfunction $(44.54 \%)$. A weak negative linear relationship was shown between adherence to lifestyle modification and the total number of diabetes complications $(r=-0.1398, p=0.1295)$ but was not significant thus linear regression was not done. Based on the study findings, we recommend that adherence to lifestyle modification should be assessed routinely at each visit using objective methods such as glycosylated haemoglobin, lipid profiles and drug levels should. Short message services and support groups should be used to promote adherence. There is also need for further research on factors influencing adherence to lifestyle modification.Formal education of Diabetes Nurses or diabetes Educators and nationwide health education may also be implemented.Limitations of the study were that data was collected through face to face interviews and only one study site was used.

\section{References}

[1]. Guariguata, L., Whiting, D. R., Hambleton, I., Beagley, J., Linnenkamp, U., \& Shaw, J. E. (2014). Global estimates of diabetes prevalence for 2013 and projections for 2035.Diabetes Research and Clinical Practice, 103(2), 137-149.

[2]. IDF. (2014). Diabetes AtlasInternational Diabetes Federation. Retrieved November 6, 2015, from https://www.idf.org/diabetesatlas

[3]. WHO. (2014). WHO | Diabetes. Retrieved September 21, 2015, from http://www.who.int/topics/diabetes_mellitus/en/ 
[4]. CDC. (2014). 2014 Statistics Report | Data \& Statistics | Diabetes | CDC. Retrieved November 2, 2015, from http://www.cdc.gov/diabetes/data/statistics/2014statisticsreport.html

[5]. Smeltzer .S.C, Bare, Bg., Hinkel, JL., Cheever, KH. (2010) Brunner and Suddarth's textbook ofMedical surgical nursing.Philadelphia, Lippin Williams and Wilkins.

[6]. Ministry of Health and Child Care, Zimbabwe (2013).Essential Drugs List for Zimbabwe. Harare.

[7]. [7]García-Pérez, L.-E., Álvarez, M., Dilla, T., Gil-Guillén, V., \& Orozco-Beltrán, D. (2013). Adherence to Therapies in Patients with Type 2 Diabetes.Diabetes Therapy, 4(2), 175-194. http://doi.org/10.1007/s13300-013-0034-y

[8]. Saleh, F., Mumu, S. J., Ara, F., Hafez, M. A., \& Ali, L. (2014). Non-adherence to self-care practices \& medication and health related quality of life among patients with type 2 diabetes: a cross-sectional study. BMC Public Health, 14(1), 1-16. http://doi.org/10.1186/1471-2458-14-431

[9]. Grave, R. D., Calugi, S., Centis, E., Marzocchi, R., Ghoch, M. E., \&Marchesini, G. (2010). Lifestyle modification in the management of the metabolic syndrome: achievements and challenges. Diabetes, Metabolic Syndrome and Obesity: Targets and Therapy, 3, 373-385. http://doi.org/10.2147/DMSOTT.S13860

[10]. Foreyt, J. P., \& Poston, W. C. (1999). The challenge of diet, exercise and lifestyle modification in the management of the obese diabetic patient.International Journal of Obesity \& Related Metabolic Disorders, 23, s5.

[11]. Patel, M., Patel, I. M., Patel, Y. M., \&Rathi, S. K. (2011). A Hospital-based Observational Study of Type 2 Diabetic Subjects from Gujarat, India. Journal of Health, Population, and Nutrition, 29(3), 265-272.

[12]. Reinehr, T. (2013). Type 2 diabetes mellitus in children and adolescents. World Journal of Diabetes, 4(6), $270-281$. http://doi.org/10.4239/wjd.v4.i6.270

[13]. IDF.(2015). Diabetes Atlas | International Diabetes Federation. Retrieved November 6, 2015, from https://www.idf.org/diabetesatlas

[14]. Ministry of Finance and Economic Development, Zimbabwe. (2014). Ministry of Finance and Economic Development - Poverty Datum Lines - January 2014. Retrieved November 6, 2015, from http://www.zimtreasury.gov.zw/189-poverty-datum-lines-january2014

[15]. Pender, N. J. (2011). Heath Promotion Model Manual. Retrieved from http://deepblue.lib.umich.edu/handle/2027.42/85350

[16]. Jin, J., Sklar, G. E., Min Sen Oh, V., \&Chuen Li, S. (2008). Factors affecting therapeutic compliance: A review from the patient's perspective. Therapeutics and Clinical Risk Management, 4(1), 269-286. 